\title{
Novel physical effects in intersecting waveguides
}

\author{
L. McCaughan and Niraj Agrawal \\ Department of Electrical and Computer Engineering, University of Wisconsin, Madison, Wisconsin 53706 \\ G. A. Bogert \\ AT\&T Bell Laboratories, 555 Union Boulevard, Allentown, Pennsylvania 18103
}

(Received 24 June 1987; accepted for publication 31 August 1987)

Previous theory of intersecting waveguides (optical) based on the idea of composite modes is unable to explain a variety of importani experimental observations. We have developed a systematic theory which is in good agreement with the experiment. In addition, our theory points out the novel physical effects associated with this geometry. It is found that the crosstalk vanishes for an intersection angle which is twice the angle of rays in the individual waveguides. A sharply peaked radiation pattern from the intersection region of the two waveguides is both predicted and observed for the first time.

A description of the interaction between two intersecting waveguides is an important problem in integrated optics. The absence of $S$ bends makes it possible to achieve higher levels of integration with electro-optical switches based on this geometry than is possible with directional couplers. ${ }^{1}$ hntersecting waveguides can also be used to make low-loss crossovers with negligible crosstalk. ${ }^{2}$ The previous theory of intersecting waveguides invokes the concept of interference between the symmetric and antisymmetric modes of this composite waveguide structure. ${ }^{3}$ Such an analysis is invalid, because the optical fields in the two waveguides do not propagate in the same direction. A multiple scattering analysis has recently been developed to describe the physics of two intersecting waveguides in terms of a direct coupling between the modes of the individual slab waveguides. ${ }^{4}$ In this picture the two waveguides interact by scattering the felds of each other in a self-consistent manner. Here, we report some novel physical effects as pointed cut by the multiple scatiering analysis of interaction between two singlemode intersecting waveguides.

The wave equation for the transverse electric (TE) mode $\left(\partial / \partial x=0, \nabla_{i}^{2} \equiv \partial^{2} / \partial y^{2}+\partial^{2} / \partial z^{2}\right)$ with a harmonic time dependence $\exp (-i \omega t)$ is reduced to

$$
\left[\nabla_{t}^{2}+\omega^{2} \mu \epsilon(y, z)\right] E_{x}(y, z)=0 .
$$

The dielectric permitivity is given by

$$
\epsilon(y, z)=\epsilon_{s}+\epsilon_{1}(y, z)+\epsilon_{2}(y, z)+\iota(y, z),
$$

where $\epsilon_{s}$ pertains to the substrate, $\epsilon_{1,2}(y, z)$ represent the two intersecting single-node waveguides, and $\iota(y, z)$ is used to incorporate an arbitrary scattering inhomogeneity in the intersection region. The total field $E_{x}(y, z)$ is decomposed in terms of fields $E_{x, i}(y, z)$ associated with the two waveguides with $i=1,2$. The latter, in turn, are expressed as a sum of fields due to an infinite sequence of scattering interactions as

$$
E_{x, i}(y, z)=\sum_{n=0}^{\infty} E_{x, i}^{(n)}(y, z)
$$

The fields in the two waveguides in the zeroth order of scattering interaction are denoted by $E_{x, 1}^{(0)}(y, z)$ and $E_{x, 2}^{(6)}(y, z)$. Since the fundamental guided mode is launched from the left of the junction at $z \rightarrow-\infty$ in waveguide 1 , we obtain

$$
\begin{aligned}
& E_{x, 1}^{(0)}(y, z)=\Psi_{1}(y, z), \\
& E_{x, 2}^{(0)}(y, z)=0,
\end{aligned}
$$

where $\Psi_{1,2}$ represent the normalized (unit power) eigenfunctions corresponding to $T E_{0}$ mode of the two slab waveguides. The $n$th order scattered fields $E_{x, i}^{(n)}(y, z)$ in the two waveguides for $n \geqslant 1$ are expressed as

$$
\begin{aligned}
E_{x, 2}^{(n)}(y, z)= & \frac{i \omega}{4} \iint\left[\epsilon_{2}\left(y^{\prime}, z^{\prime}\right)+\ell\left(y^{\prime}, z^{\prime}\right)\right] \\
& \times E_{x, 1}^{(n-1)}\left(y^{\prime}, z^{\prime}\right) G_{2}\left(y, z^{\prime} ; y^{\prime}, z^{\prime}\right) d y^{\prime} d z^{\prime}, \\
E_{x, 1}^{(n)}(y, z)= & \frac{i \omega}{4} \iint\left[\epsilon_{1}\left(y^{\prime}, z^{\prime}\right)+\iota\left(y^{\prime}, z^{\prime}\right)\right] \\
& \times E_{x, 2}^{(n)}\left(y^{\prime}, z^{\prime}\right) G_{1}\left(y, z ; y^{\prime}, z^{\prime}\right) d y^{\prime} d z^{\prime},
\end{aligned}
$$

where the Green's function $G_{i}\left(y, z ; y^{\prime}, z^{\prime}\right)$ for the two slab waveguides with $i=1,2$ is defined by

$\left[\nabla_{i}^{2}+\omega^{2} \mu\left(\epsilon_{s}+\epsilon_{i}\right)\right] G_{i}\left(y, z ; y^{\prime}, z^{\prime}\right)=i 4 \omega \mu \delta\left(y-y^{\prime}, z-z^{\prime}\right)$.

The Green's function is obiained in its Fourier integral representation in the complex wave number plane which contains poles as well as branch points. The poles and branch points correspond to guided and radiation modes of the two slab waveguides, respectively. Unlike the paraliel waveguides in directional couplers, it is possible for the guided modes in one of the two intersecting waveguides to be momentum matched to the radiation modes in the other (see Fig. 1). Thus coupling of guided modes to the radiation modes is an essential and unique feature of interaction between two intersecting waveguides.

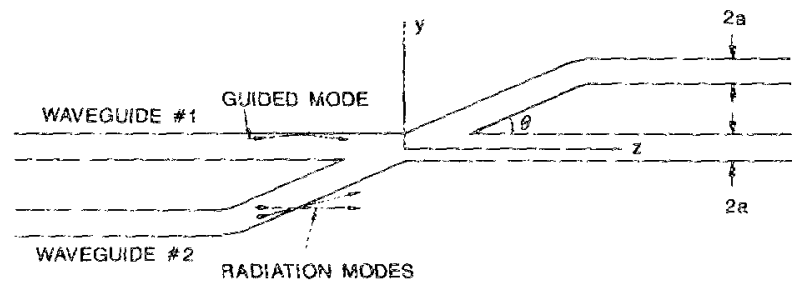

FTG. 1. Schematic diagram of guided and radiation modes in the geometry of intersecting waveguides with attached $S$ bends. The guided and radiation modes of the individual waveguides as depicted are momentum matched. 
The coupling between fundamental guided modes of the two intersecting slab waveguides can be obtained by substituting the appropriate Green's functions in $\mathrm{Eq}$. (5). It is shown that the Green's function corresponding to the guided mode for the two single-mode waveguides with $i=1,2$ can be expressed in terms of their eigenfunctions as

$$
G_{i}\left(y, z ; y^{\prime}, z^{\prime}\right)=\Psi_{i}^{\prime}\left(y^{\prime} z^{\prime}\right) \Psi_{i}(y, z) .
$$

At small $\left(\leqslant 1^{\circ}\right)$ intersection angles, the two intersecting waveguides interact strongly because of an enhanced overlap of the fields. The contribution from all orders of scattering interaction must be evaluated in such a situation because the strong interaction corresponds to several coupling lengths. Substituting Eqs. (4)-(6) in Eq. (3), the power transferred from waveguide 1 to 2 converges to ${ }^{4}$

$$
p_{x} \approx \sin ^{2}\left(K_{0}\right)
$$

where the integrated coupling coeflicient $K_{0}$ is given by the overlap integral of fields in the two waveguides as

$$
\begin{aligned}
K_{0}= & \frac{\omega}{4} \int_{-\infty}^{\infty} \int_{-\infty}^{\infty}\left[\epsilon_{2}\left(y^{\prime}, z^{\prime}\right)+i\left(y^{\prime}, z^{\prime}\right)\right] \\
& \times \Psi_{1}\left(y^{\prime}, z^{\prime}\right) \Psi_{2}^{*}\left(y^{\prime}, z^{\prime}\right) d y^{\prime} d z^{\prime} .
\end{aligned}
$$

It is found from Eqs. (7a) and (7b) that the dependence of light coupled from one waveguide to the other as anction of intersection angle is oscillatory. The period of oscillation is found to increase with the interaction angle. Such a response has been experinentally observed and motivated the earlier analyses based on the idea of interference between modes of the composite structure. ${ }^{3,5}$

At large $\left(\gtrsim 3^{\circ}\right)$ intersection angles, coupling between the two intersecting waveguides is greatly reduced. In such a case, a single scattering event (Born approximation) is sufhcient to evaluate the interaction between the two waveguides. The expression for crosstalk between the two waveguides becomes

$$
p_{x} \approx\left|K_{0}\right|^{2}
$$

and is plotted as a function of intersection angle in Fig. 2. The waveguide parameters, namely, halfwidth $a=2 \mu \mathrm{m}$, substrate index $n_{s}=2.300$, and film index $n_{f}=2.305$ are selected for numerical analysis throughout this letter so as to obtain single-mode waveguides in thium niobate at a wavelength $\lambda=1.3 \mu \mathrm{m}$. At this stage, it should be pointed out

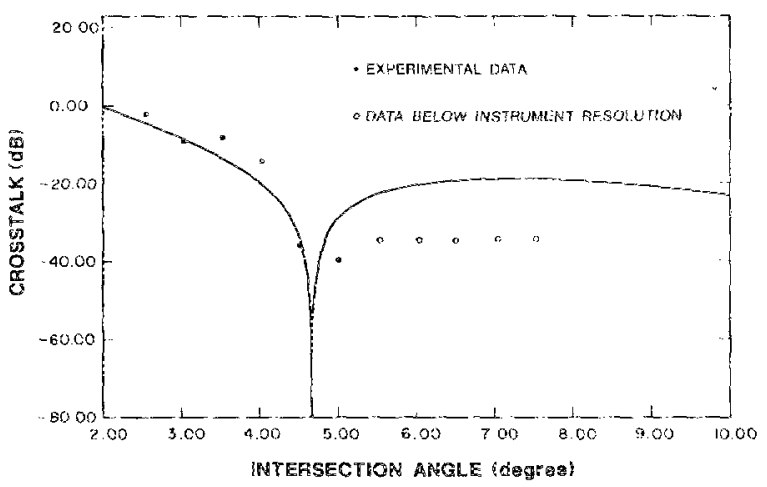

FIG. 2. Plot of calculated and measured crosstalk as 2 function of intersection angle for single $\Delta t$ (i.e., a uniform index everywhere in the guiding region) intersecting waveguides.

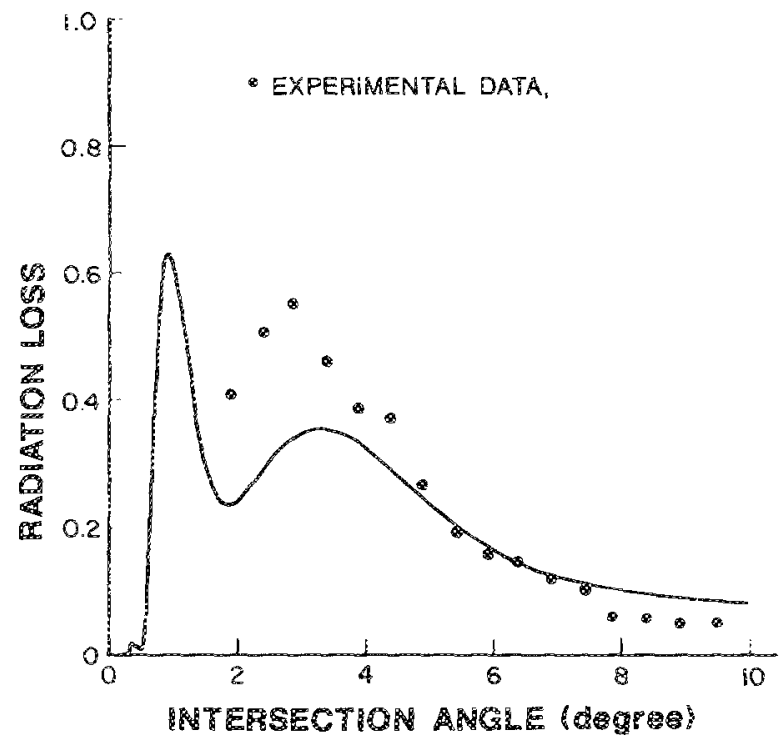

HIG. 3. Calculated and measured radiation loss as a function of intersection angle for single- $\Delta n$ insersecting waveguides. The calculated radiation loss is normalized with respect to the incident power.
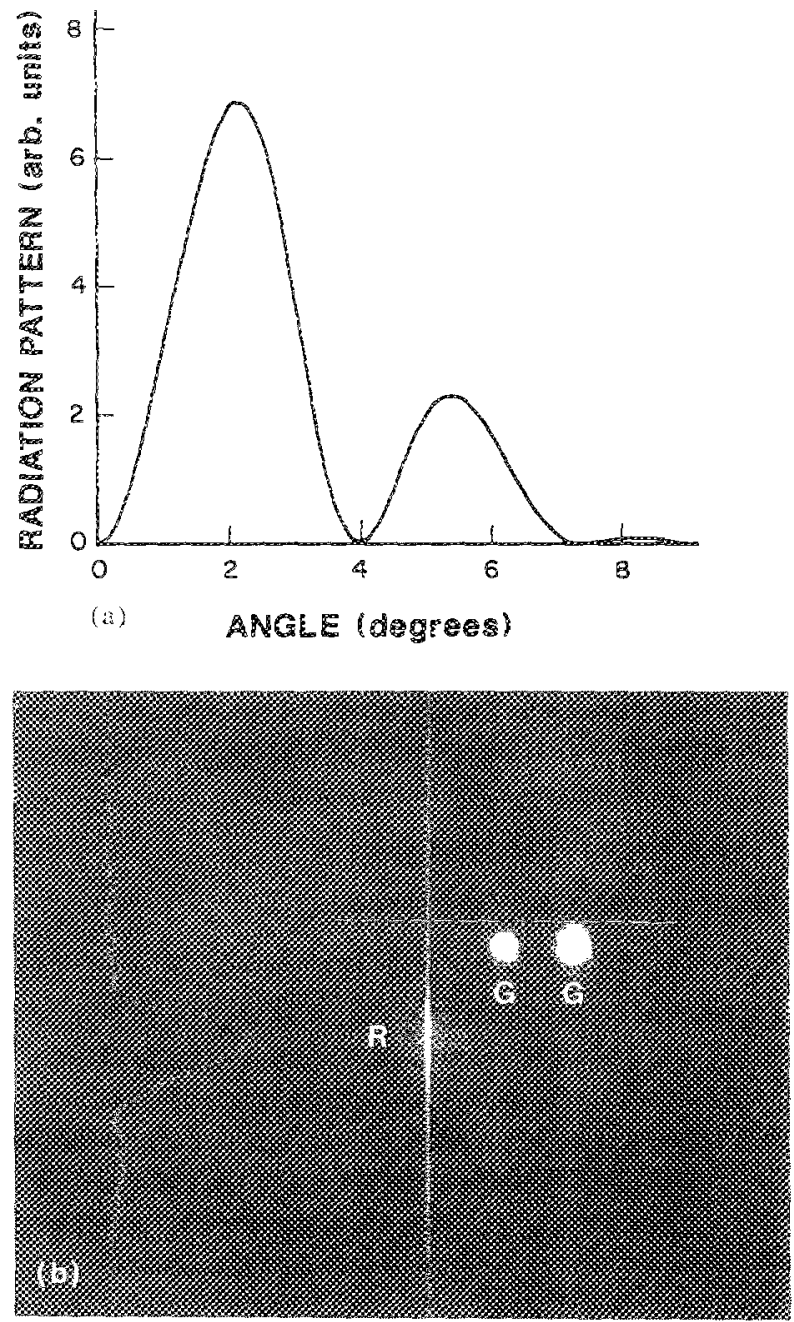

FIG. 4. (a) Calculated radiation pattern (angle measured relative to the axis of waveguide 2 ). (b) Photograph of the observed guided $(G)$ and radiated $(\mathbb{R})$ light from the junction of two waveguides intersecting at an angle of $3^{2}$. The vertical line through the radiated beam represents the cursor of the detector used to scan the intensity profilt shown at the extreme left. The approximate position of air- $\mathrm{LNBO}_{3}$ interface is indicated by a dashed horzontal line. 
that the convention for classifying transverse electric and magnetic modes for a slab and a channel wayeguide is different. Therefore, the TE (TM) modes of a slab waveguide are physically equivalent to the TM (TE) modes of a channel waveguide. As seen in this figure, the crosstalk vanishes for an intersection angle which is shown to be equal to twice the angle of rays in the individual waveguides. Experimental results from $\mathrm{T}$ :LiNbO $\mathrm{L}_{3}$ intersecting channel waveguides, ${ }^{2}$ plotted in the same figure, are in good guantitative agreement with the theory.

The coupling of guided-to-radiation modes is calculated by substituting in Eq. (5) the Green's function correspond ing to the continuous spectrum. The Poynting vector is then calculated in the Horn approximation and is integrated over a closed surface enclosing intersecting waveguides. A more detailed account of this analysis will be reported elsewhere." The coupling of guided-to-radiation modes leads to a sharply peaked radiation pattern, which in tum is responsible for the anomalous loss characteristics observed in single $A$ in intersecting waveguides. ${ }^{2}$ The calculated radiation loss as a function of intersection angle is in good agreement with the measurements of insertion loss (see Fig. 3). In Fig. 4(a) the calculated ratiation patten is plotted for two waveguides intersecting at an angle of $3^{\circ}$ for which the radiation loss is a local maximum. The experimental observation of such a pattern is shown in Eig. 4 (b). Note that a pronounced radiation beam appears below the surface of the LibO, substrate. The downward deflection of radiation beam occurs because of additional mode confinement in verticat direction in the channel waveguide geometry. A simple analysis which takes into account the effect of attached 5 bends to the intersecting waveguides (cf. Fig. 1 ) yields an angular position of the radiated beam to be $2.2^{\circ}$ relative to waveguide 2 . This value is in good agreement with the position of the main lobe in the radiation patem predicted by theory and shown in Fig. 4(a). It is important to note that a good agreement is oblained with no systematic attempt to fit theory (siab-TE mode) to the experimental data (channel-TV mode).

In summary, we have developed a systematic theory which not only provides an interpretation for all the experimental observations, but also points out the novei physical effects in intersecting waveguides.

This research was supported by the National Science Foundation under grants ECS-8504513 and ECS-8508024.

${ }^{i L}$ McCiughan, Opt. Eng. 24, 241 (1985).

${ }^{2}$ G. A. Bogert, Electron. Lett. 23, 72 (1987),

${ }^{3}$ A. Neyer, W. Meverkamp, Thylen, and 1 . Lagerströn, J. Lightwave Tectincl. ET-3, 635 (1985)

${ }^{4}$ N. Agrawal, L. McCaughan, and S. R. Seshadri, J. Appl. Phys. 62. 2187 (1987).

${ }^{5}$ F. E. Bergmann, L. McCaughan, and J. E. Warson, Appl. Opt. 23, 3000 (1984).

N. Agrawal and L. McCaughan (umpubished). 